This item was submitted to Loughborough's Research Repository by the author.

Items in Figshare are protected by copyright, with all rights reserved, unless otherwise indicated.

\title{
Pain-related evoked potentials are modulated across the cardiac cycle
}

PLEASE CITE THE PUBLISHED VERSION

http://dx.doi.org/10.1016/j.pain.2007.10.010

\section{PUBLISHER}

Elsevier / @ International Association for the Study of Pain

VERSION

AM (Accepted Manuscript)

LICENCE

CC BY-NC-ND 4.0

\section{REPOSITORY RECORD}

Edwards, Louisa, Koji Inui, Christopher Ring, Xiaohong Wang, and Ryusuke Kakigi. 2019. "Pain-related Evoked Potentials Are Modulated Across the Cardiac Cycle”. figshare. https://hdl.handle.net/2134/15381. 
This item was submitted to Loughborough's Institutional Repository (https://dspace.lboro.ac.uk/) by the author and is made available under the following Creative Commons Licence conditions.

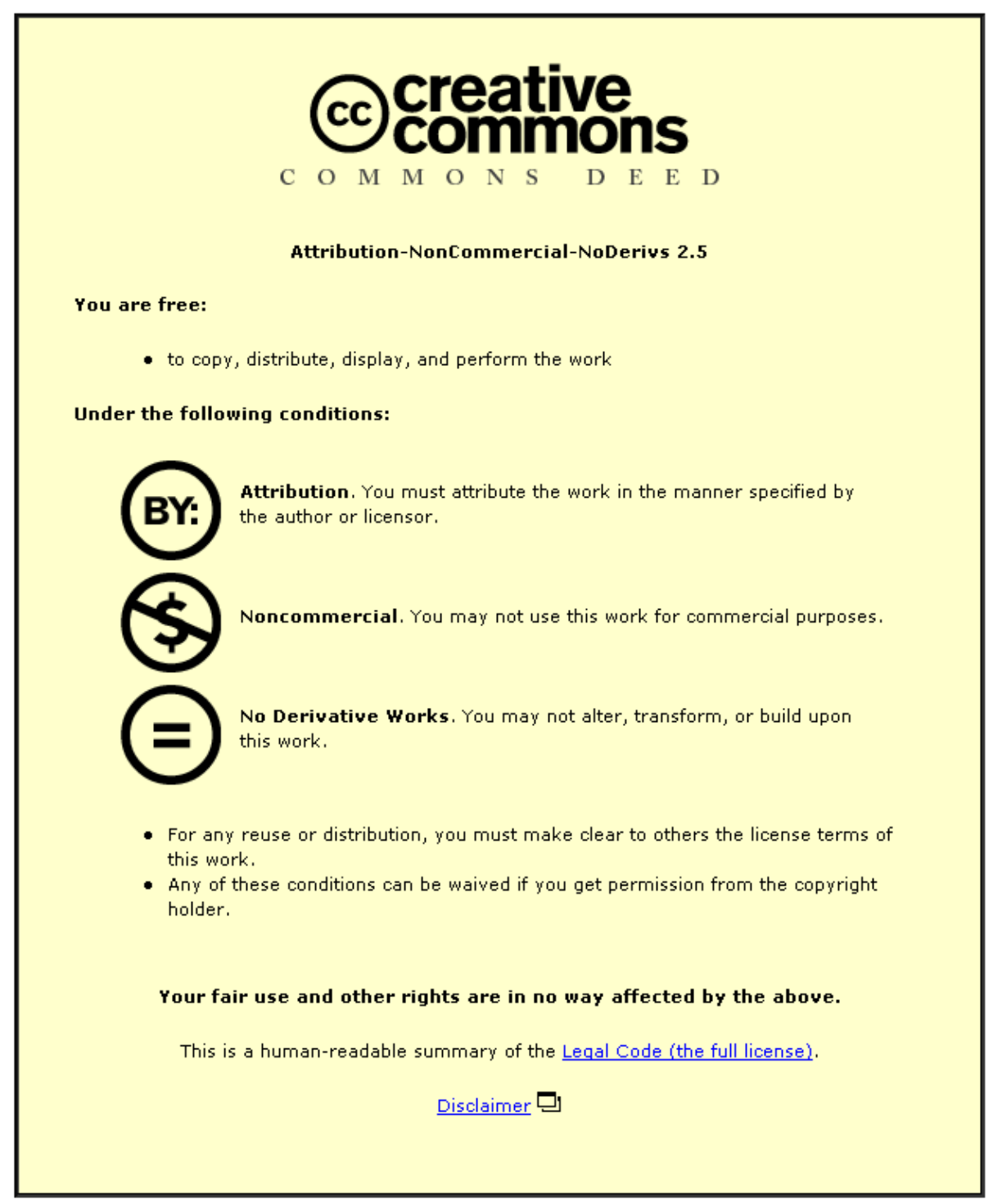

For the full text of this licence, please go to: http://creativecommons.org/licenses/by-nc-nd/2.5/ 


\section{Pain-related evoked potentials are modulated across the cardiac cycle}

Louisa Edwards ${ }^{1}$, Koji Inui ${ }^{2}$, Christopher Ring ${ }^{1}$, Xiaohong Wang ${ }^{2}$, Ryusuke Kakigi ${ }^{2,3}$

${ }^{1}$ International Centre for Health and Exercise Research, University of Birmingham, Birmingham. B15 2TT. UK.

${ }^{2}$ Department of Integrative Physiology, National Institute for Physiological Sciences, Okazaki 444-8585, Japan.

${ }^{3}$ RISTEX, Japan Science and Technology Agency.

Text pages: 30

Figures: 6

Tables: 0

Corresponding Author: Dr Louisa Edwards, International Centre for Health and Exercise Research, University of Birmingham, Birmingham. B15 2TT. United Kingdom. Telephone: +44 121415 8785, Facsimile: +44 121414 4121, E-mail:

L.Edwards@bham.ac.uk 


\begin{abstract}
Evidence suggests that the arterial baroreceptors modulate pain. To examine whether cortical processing of nociception is modulated by natural variations in arterial baroreceptor stimulation during the cardiac cycle, peak-to-peak amplitudes of the N2-P2 pain-related potential and pain ratings were recorded in response to noxious laser stimulation at different times during the cardiac cycle in 10 healthy males. Significant variations in the N2-P2 amplitudes occurred across the cardiac cycle, with smaller amplitudes midcycle, indicating that cortical processing of nociception was attenuated during systole compared to diastole. Pain ratings did not vary across the cardiac cycle. These data support the hypothesis that arterial baroreceptors modulate the processing of nociception during each cardiac cycle.
\end{abstract}

Keywords: Arterial baroreceptors; Cardiac cycle; Pain ratings; Pain-related evoked potentials 


\section{Introduction}

The arterial baroreceptors are stretch receptors located in the aortic arch and carotid sinus that are naturally stimulated during systole by distension of the arterial wall by the pressure pulse wave (Mancia and Mark, 1983). Baroreceptor activation has been shown to inhibit sensory (Gahery and Vigier, 1974) and motor (Koch, 1932) processes. Mounting evidence indicates that pain and nociception also vary with baroreceptor activity. Using the nociceptive flexion reflex, a polysynaptic spinal reflex that facilitates withdrawal from noxious stimuli to avoid tissue injury (Sandrini et al., 2005), a series of studies found that nociception was attenuated during systole, when the baroreceptors are most active, compared to diastole (Edwards et al., 2001;Edwards et al., 2002;Edwards et al., 2003;Al'Absi et al., 2005;McIntyre et al., 2006). In contrast, concurrent pain ratings did not vary across the cardiac cycle (Edwards et al., 2001;Edwards et al., 2002;Edwards et al., 2003). However, pain was attenuated when the carotid baroreceptors were artificially stimulated, beyond the normal physiological range, by neck suction (for review see Rau and Elbert, 2001).

Studies have also examined the effects of neck suction on pain-related evoked brain potentials comprising a negativity (N2) followed by a positivity (P2). These potentials correlate with both pain reports and stimulus intensity (Bromm and Meier, 1984) and are attenuated by centrally-acting analgesics (Scharein and Bromm, 1998), and therefore, have been interpreted as reflecting the cognitive processing of a noxious stimulus (Handwerker and Kobal, 1993). Both N2 and P2 amplitudes (Mini et al., 1995) 
and the peak-to-peak N2-P2 amplitude (Angrilli et al., 1997) elicited by noxious intracutaneous electrical stimulation of the finger were found to be attenuated by neck suction. However, another study has reported that the N2-P2 amplitude was augmented by neck suction (Brody et al., 1997). Accordingly, these studies indicate that stimulation of the arterial baroreceptors can modulate processing of noxious stimuli.

To date, no studies have investigated whether natural variations in baroreceptor stimulation across the cardiac cycle, in the normal physiological range, influence cortical processing of noxious stimuli. The current study investigated the influence of the cardiac cycle, as an index of pulsatile variations in blood pressure, on the cortical processing of nociception. The study used thulium-evoked laser stimulation, that exclusively activates nociceptive nerve fibres, to evoke pain-related late brain potentials (Mor and Carmon, 1975;Kakigi et al., 1989). Based on previous findings that the nociception flexion reflex is attenuated during systole, it was hypothesised that N2-P2 amplitude, an objective index of the degree of induced pain (Bromm and Lorenz, 1998), would be smaller during systole than diastole.

\section{Methods}

\subsection{Participants}

Ten healthy male normotensive volunteers, with a mean age of 33 years $(S D=$ 6), mean height of $171 \mathrm{~cm}(S D=4)$ mean weight of $65 \mathrm{~kg}(S D=6)$, mean systolic blood 
pressure of $120 \mathrm{mmHg}(S D=11)$, mean diastolic blood pressure of $77 \mathrm{mmHg}(S D=9)$ and mean heart rate of $63 \mathrm{bpm}(S D=11)$, participated in the study. All participants were free from neurologic and psychiatric diseases and psychiatric and analgesic medications. Participants were asked to refrain from alcohol, caffeine and smoking for at least 12 hours prior to testing. The study was approved by the Ethics Committee at National Institute for Physiological Sciences, Okazaki; all volunteers gave informed consent to participate.

\subsection{Laser stimulation}

A thulium:YAG laser stimulator (Carl Baasel Lasertech, Starnberg, Germany) was used to produce noxious stimuli. Laser pulses (1 ms in duration, $2000 \mathrm{~nm}$ in wavelength, and $3 \mathrm{~mm}$ in spot diameter) were delivered to the dorsum of the right hand at an interval of between 15 and $20 \mathrm{~s}$. The irradiated points were moved slightly for each stimulus to avoid tissue damage and habituation of the receptors. At the start of the session, 10-20 laser stimuli were delivered to determine the stimulus intensity required to produce a painful sensation. After each stimulus, the participants rated the stimulus using a visual analogue scale (VAS), with anchors of 0 (no painful sensation) and 100 (imaginary intolerable pain sensation). A stimulus intensity $(M=158, S D=9 \mathrm{~mJ})$, rated as approximately 50 on the VAS, was used to examine pain-related evoked potentials (see below). At this laser intensity, all subjects rated the stimulus as a pricking pain sensation. Trained subjects can discriminate the first and second pain sensations, 
however, no subjects in this study reported a sensation other than pricking.

\subsection{Laser evoked potential recording}

The laser evoked potentials were recorded with an Ag/AgCl disk electrode placed over $\mathrm{Cz}$ (vertex), referred to the linked earlobes (A1+A2) of the International 10/20 System. A pair of electrodes placed on the supra- and infra-orbit of the right eye was used for recording an electro-oculogram. An electrocardiogram was recorded using a pair of disk electrodes placed on each forearm. The impedance of all electrodes was kept below $5 \mathrm{k} \Omega$. The electroencephalographic signals were recorded with a $0.1 \mathrm{~Hz}$ to $100 \mathrm{~Hz}$ bandpass filter and digitized at a sampling rate of $1000 \mathrm{~Hz}$. The period of analysis was $800 \mathrm{~ms}$ before to $600 \mathrm{~ms}$ after stimulus onset; the pre-stimulus period was used as the DC baseline. Individual trials containing artifacts due to eye blinks were rejected before averaging.

\subsection{Procedure}

Each subject was seated in an armchair in a quiet, electrically shielded, and temperature controlled (24 to $26^{\circ} \mathrm{C}$ ) room. Laboratory systolic blood pressure (mmHg), diastolic blood pressure (mmHg), and heart rate (bpm) were measured three times using a mercury sphygmomanometer and a brachial cuff attached to the participant's upper left arm. The experimental session consisted of 5 blocks of 12 trials. Each block was 
separated by a 10 minute rest period. During the experiment, a fixation point (a white circle $2 \mathrm{~cm}$ in diameter) was displayed on a screen $1.5 \mathrm{~m}$ in front of the subjects from 10-15 s before until $2 \mathrm{~s}$ after each stimulus. Subjects were instructed to look at the fixation point when it was displayed. Two seconds after the onset of each stimulus, the fixation point disappeared and 'VAS' was displayed for three seconds, during which subjects rated the perceived sensation. Then the fixation point appeared again to prepare the next stimulus. The participants were instructed to rate the perceived pricking sensation associated with each laser stimulation by marking a $100 \mathrm{~mm}$ VAS.

\subsection{Data reduction and analysis}

The R-wave latency relative to stimulus onset (ms) and peak-to-peak amplitude $(\mu \mathrm{V})$ of the N2-P2 component were measured in each trial. The peak of N2 and P2 was determined during a latency period of 180-240 ms and 280-400 ms, respectively, for each trial. To show the variability of N2/P2 components in each trial, the waveforms of 12 consecutive trials in a representative participant are depicted in Figure 1. In addition, the amplitudes of each N2 and P2 component were measured, using a DC offset, from the prestimulus baseline of $-100 \mathrm{~ms}$ to the peak negativity and positivity, respectively. Trials were then sorted into one of eight $100 \mathrm{~ms}$ wide intervals (each interval is labeled by its midpoint), whose minimum and maximum indicated the timing of the noxious stimulation after the R-wave: 0-99 ms (R+50 ms), 100-199 ms (R+150 ms), 200-299 ms (R+250 ms), 300-399 ms (R+350 ms), 400-499 ms ( +450 
ms), 500-599 ms (R+550 ms), 600-699 ms (R+650 ms) and 700-800 ms (R+750 ms). The mean (SD) number of trials per R-wave to stimulation interval was 5.0 (1.6), 5.3 (2.8), 6.3 (2.8), 5.4 (2.8), 5.4 (1.8), 6.2 (1.9), 5.4 (2.9), 6.4 (2.2) for R-wave intervals R + 50 to $\mathrm{R}+750 \mathrm{~ms}$, respectively. All participants provided data for every R-wave to stimulation interval. Data were lost (25\% of total number of trials) on trials with blink artifacts and trials when the R-wave occurred more than 800 ms before the onset of noxious stimulation. The mean N2-P2 peak-to-peak amplitudes $(\mu \mathrm{V})$ and pain ratings were calculated for each R-wave to stimulation interval. Repeated measures analyses of variance (ANOVAs) with $R$-wave to stimulation interval (i.e., $R+50, R+150, R+250$, $\mathrm{R}+350, \mathrm{R}+450, \mathrm{R}+550, \mathrm{R}+650, \mathrm{R}+750 \mathrm{~ms}$ ) as a within-subjects factor were performed on the N2-P2 amplitudes and pain ratings. ANOVAs were corrected for the assumption of independence of data points using the Huynh-Feldt correction $(\varepsilon)$. Eta-squared $\left(\eta^{2}\right)$, a measure of effect size, is also reported. A significance level of .05 was adopted.

Significant results were followed by LSD post hoc tests. The data were analyzed using Statistica '99.

\section{Results}

\subsection{N2-P2 peak-to-peak amplitudes}

A repeated measures ANOVA (8 Intervals) revealed significant variations in the N2-P2 amplitude across the cardiac cycle, $\varepsilon=.74, F(7,63)=3.15, p=.02, \eta^{2}=.26$, 
which were characterized by a quadratic trend, $F(1,9)=29.83, p=.0005, \eta^{2}=.77$, (see Figure 2). Post-hoc comparisons confirmed that the N2-P2 amplitudes elicited by stimulation at $\mathrm{R}+250, \mathrm{R}+350$ and $\mathrm{R}+450$ ms were smaller than those elicited at $\mathrm{R}+50$, $\mathrm{R}+150$ and $\mathrm{R}+750 \mathrm{~ms}$. For illustrative purposes, the grand mean waveforms, averaged for the early $(R+50, R+150 \mathrm{~ms})$, middle $(\mathrm{R}+250, \mathrm{R}+350, \mathrm{R}+450 \mathrm{~ms})$ and late $(\mathrm{R}+550$, $\mathrm{R}+650, \mathrm{R}+750 \mathrm{~ms}$ ) phases of the cardiac cycle are presented in Figure 3, where it can be seen that the amplitudes were smaller mid-cycle compared to early and late cycle.

\subsection{N2 amplitudes}

A repeated measures ANOVA (8 Intervals) revealed significant variations in the N2 amplitude across the cardiac cycle, $\varepsilon=.99, F(7,63)=4.13, p=.001, \eta^{2}=.31$, which were characterized by a quadratic trend, $F(1,9)=25.43, p=<.001, \eta^{2}=.74$, (see Figure 4). Post-hoc comparisons confirmed that the N2 amplitudes elicited by stimulation at $\mathrm{R}+250$ ms was smaller than $\mathrm{R}+50, \mathrm{R}+150, \mathrm{R}+650$ and $\mathrm{R}+750$ ms. Stimulation at $\mathrm{R}+350$ ms produced smaller $\mathrm{N} 2$ amplitudes than $\mathrm{R}+150, \mathrm{R}+650$ and R+750 ms. Finally, stimulation at R+450 ms produced smaller N2 amplitudes than $\mathrm{R}+650$ and $\mathrm{R}+750 \mathrm{~ms}$.

\subsection{P2 amplitudes}

A repeated measures ANOVA (8 Intervals) did not reveal significant 
variations in the $\mathrm{P} 2$ amplitude across the cardiac cycle, $\varepsilon=.84, F(7,63)=0.73, p=.63$, $\eta^{2}=.07$, (see Figure 5).

\subsection{Pain ratings}

A repeated measures ANOVA (8 Intervals) revealed no significant differences in pain ratings across the cardiac cycle, $\varepsilon=.64, F(7,63)=1.10, p=.37, \eta^{2}=.11$ (see Figure 6).

\section{Discussion}

The present study found significant variations across the cardiac cycle in the amplitude of the N2-P2 pain-related components of the evoked potential elicited by noxious laser stimulation. The N2-P2 amplitude difference is believed to be an objective index of the degree of induced pain (Bromm and Lorenz, 1998). Indeed, positive relationships have been found between the intensity of noxious laser stimuli, the amplitude of the N2-P2, and the magnitude of pain sensation (Bromm, 1991). The observation of smaller amplitude N2-P2 waveforms during the middle of the cardiac cycle indicates that pain-related cortical responses were attenuated during systole compared to diastole. Accordingly, these data support the hypothesis that stimulation of the arterial baroreceptors by natural changes in blood pressure during the cardiac cycle has a dampening effect on the nociceptive system. 
In the present study, we only recorded the N2-P2 components of the evoked potential from one electrode at $\mathrm{Cz}$. Therefore, the data cannot reveal the precise mechanisms of N2-P2 modulation across the cardiac cycle. However, the grand-averaged waveform (see Fig. 3) suggests that the cardiac cycle effect was larger for N2 than P2. Indeed, separate analyses of the N2 and P2 components revealed cardiac cycle time effects for N2 and not P2. The N2 and P2 components are generated mainly in the anterior cingulate cortex (Tarkka and Treede, 1993;Bromm and Chen, 1995; Valeriani et al., 1996). In addition to anterior cingulate cortex, the secondary somatosensory cortex or insula cortex contribute to shape the N2 component (Tarkka and Treede, 1993;Bromm and Chen, 1995; Valeriani et al., 1996;Peyron et al., 2002). Therefore, our findings are compatible with the hypothesis that the target site of the interaction between N2-P2 and baroreceptor output is the somatosensory or insula cortex. Further studies employing multi-channel recordings are required to test this hypothesis.

This is the first study, to our knowledge, to describe modulation of the pain-related evoked potential with natural variations in baroreceptor activation across the cardiac cycle. The current findings broadly agree with previous research which has reported reduced N2-P2 amplitudes elicited by intracutaneous stimulation of the finger during artificial stimulation of the baroreceptors using neck suction (Mini et al., 1995;Angrilli et al., 1997). In addition, the current data are in line with reports of dampened lower limb nociceptive flexion reflex responding during systole compared to diastole (Edwards et al., 2001;Edwards et al., 2002;Edwards et al., 2003;McIntyre et al., 
2006). The modulating effect of the cardiac cycle on the brain appears not to be exclusive to nociception. Auditory and visual perception vary with the phase of the cardiac cycle: responses are generally slowest at the start of the cardiac cycle and quicken as the cycle progresses (Saxon, 1970;Sandman et al., 1977). Further, modulation of visual and auditory event-related potentials has been demonstrated during systole and diastole: the P1 component of the visual evoked potential (Walker and Sandman, 1982) and the N1 component of the auditory evoked potential (Sandman, 1984) were smaller during systole. Previous research has demonstrated that rhythmic oscillations of the EEG, most notably in the alpha range, were time locked to the carotid pressure wave (Walker and Walker, 1983). Other research has examined the effects of artificial baroreceptor stimulation on the brain. A classic study in cats showed that mechanical stimulation of the carotid sinus baroreceptors had an inhibitory influence on cortical excitability (Bonvallet et al., 1954). Further, artificial baroreceptor stimulation in humans has been shown to cause a substantial reduction in slow cortical negative potentials, particularly the contingent negative variation, an index of cortical arousal (Rau et al., 1988;Elbert et al., 1988;Rau et al., 1993). Accordingly, the current cycle time effect for the pain-related evoked potential adds to a compelling body of evidence for a relationship between the cardiovascular system and the brain.

Pain was not modulated across the cardiac cycle in the current study. This is in line with previous studies which found no differences in pain reports for electrocutaneous stimuli delivered at various intervals after the R-wave of the electrocardiogram (Edwards et al., 2001;Edwards et al., 2002;Edwards et al., 2003). 
These findings contrast with the results of other studies that employed artificial baroreceptor manipulations. These studies reported that pain was lower during systole compared to diastole during neck suction (Al'Absi et al., 2005), during repeated neck suction and compression (Rau et al., 1994;Mini et al., 1995), as well as during single neck suction and compression pulses (Edwards et al., 2003). These contradictory findings may be due to differences between natural and artificial baroreceptor stimulation studies in terms of the level of baroreceptor stimulation achieved.

The mechanism by which pain-related cortical processing is attenuated by the cardiac cycle has yet to be determined. However, it is reasonable to assume that these effects might be due to natural fluctuations in arterial baroreceptor activity across the cardiac cycle (see Edwards et al., 2001;Edwards et al., 2007). In sum, the integrated baroreceptor output of aortic baroreceptors located in the aortic arch and carotid sinus can be estimated to extend from 90-390 ms after the R-wave. The current study found that the N2-P2 amplitude was attenuated when noxious stimuli were delivered to the hand during the 200-299 ms, 300-399 ms and 400-499 ms intervals after the R-wave. The onset latency of cortical activity in SI and SII, the proposed site of interaction, following noxious YAG laser stimulation to the hand has been recorded at 90-110 ms (Nakata et al., 2004;Wang et al., 2007). Thus, as N2-P2 was modulated from 200 ms after the R-wave, the earliest time the SII must be affected by baroreceptor activity is 290 ms after the R-wave. Accordingly, the observed pattern of modulation of the N2-P2 amplitude is compatible with the pattern of baroreceptor activation when a sensory transduction and processing delay of approximately $150 \mathrm{~ms}$ is included. This $150 \mathrm{~ms}$ 
delay may be explained by neural transmission times within the brainstem. For example, electrical stimulation of baroreceptor afferents in dogs and cats have been shown to cause inhibition of sympathetic activity with a latency of 150-200 ms, dependent of the recording site at the spinal level (Richter et al., 1970;Coote et al., 1981). Allowing 10-15 ms for transmission of nerve impulses from carotid sinus and aortic arch to the nucleus of the solitary tract (Seller and Illert, 1969), and approximately $30 \mathrm{~ms}$ from the rostral ventrolateral medulla to sympathetic preganglionic neurons (Mcallen, 1986), this leaves $100-150 \mathrm{~ms}$ for transmission in the lower brainstem from the nucleus of the solitary tract to the rostral ventrolateral medulla (Dembowsky and Mcallen, 1990). This 100-150 ms transduction latency could perhaps explain the 150 ms delay between baroreceptor activation and attenuation of the N2-P2 amplitudes found in the current study. Further, there is substantial evidence suggests that structures involved in the baroreflex pathway could also influence the pain system (for review see Ghione, 1996). For example, stimulation of the nucleus of the solitary tract induces antinociception (Aicher and Randich, 1990) and the A5 cell group and locus coeruleus are sources of descending noradrenergic fibers that modulate spinal nociceptive transmission (Miller and Proudfit, 1990). Furthermore, other evidence shows that pain areas are involved in baroreflex control. The periaqueductal grey matter, which produces analgesia when stimulated, can modulate the arterial baroreflex (Inui et al., 1994). The nucleus raphe magnus in the rostral ventrolateral medulla, which plays a role in pain modulation, is involved in the baroreflex pathway mentioned above, and also contains neurons that respond to noxious stimuli that show spontaneous fluctuations in phase with both 
natural variations and experimentally-induced changes in blood pressure (Thurston and Randich, 1992; Thurston and Randich, 1995). Accordingly, this evidence demonstrates a close integration of areas involved in pain modulation and cardiovascular regulation.

The current study should be interpreted in light of some possible limitations. Neither blood pressure nor vessel diameter were measured during laser stimulation. Accordingly, the extent to which the pulse pressure wave distended the aortic arch and carotid sinus was not characterized, and therefore, the precise timing and magnitude of arterial baroreceptor stimulation is not known. Further, respiration was not measured in the current study and therefore the potential moderating effects of the phase of the respiratory cycle on the effects observed across the cardiac cycle were not determined. Given that baroreceptor function can vary between inspiration and expiration (Eckberg and Sleight, 1992), research is needed to explore these putative effects. The sample size may be considered a potential weakness. However, many pain-related evoked potential studies test similar numbers of participants. The study only tested men and therefore the generalizability of the cycle time effect for the N2-P2 amplitude needs to be determined in female participants. Accordingly, firm conclusions regarding the influence of baroreceptor activation on pain-related cortical processing should not be drawn until the current findings have been replicated by larger studies of mixed gender.

In conclusion, variations in the N2-P2 amplitudes across the cardiac cycle, with smaller amplitudes mid-cycle, indicated that cortical processing of nociception was attenuated during systole compared to diastole. These data support the hypothesis that arterial baroreceptors modulate the processing of nociception during each cardiac cycle. 


\section{Reference List}

Aicher SA, Randich A. Antinociception and cardiovascular responses produced by electrical stimulation in the nucleus tractus solitarius, nucleus reticularis ventralis, and the caudal medulla. Pain 1990; 42:103-119.

Al'Absi M, France CR, Ring C, France J, Harju A, McIntyre D, Wittmers LE. Nociception and baroreceptor stimulation in hypertension-prone men and women. Psychophysiology 2005; 42:83-91.

Angrilli A, Mini A, Mucha RF, Rau H. The influence of low blood pressure and barorecepter activity on pain responses. Physiology and Behavior 1997; 62:391-397.

Bonvallet M, Dell P, Hiebel G. Tonus sympathique et activite electrique corticale. Electroencephalography and clinical Neurophysiology 1954; 6:119-144.

Brody S, Angrilli A, Weiss U, Birbaumer N, Mini A, Veit R, Rau H. Somatotosensory evoked potentials during baroreceptor stimulation in chronic low back pain patients and normal controls. International Journal of Psychophysiology 1997; 25:201-210.

Bromm B, Lorenz J. Neurophysiological evaluation of pain. Electroencephalography and clinical Neurophysiology 1998; 107:227-253.

Bromm B. Laser-evoked cerebral potentials in the assessment of cutaneous pain 
sensitivity in normal subjects and patients. Revue Neurologique 1991; 147:625-643.

Bromm B, Chen ACN. Brain Electrical Source Analysis of Laser Evoked-Potentials in Response to Painful Trigeminal Nerve-Stimulation. Electroencephalography and clinical Neurophysiology 1995; 95:14-26.

Bromm B, Meier W. The intracutaneous stimulus - A new pain model for algesimetric studies. Methods and Findings in Experimental and Clinical Pharmacology 1984; 6:405-410.

Coote JH, Macleod VH, Fleetwoodwalker SM, Gilbey MP. Baroreceptor inhibition of sympathetic activity at a spinal site. Brain Research 1981; 220:81-93.

Dembowsky K, Mcallen RM. Baroreceptor inhibition of subretrofacial neurons Evidence from intracellular-recordings in the cat. Neuroscience Letters 1990; 111:139-143.

Eckberg DL, Sleight P. Human baroreflexes in health and disease. Oxford: Clarendon Press, 1992.

Edwards L, McIntyre D, Carroll D, Ring C, France CR, Martin U. Effects of artificial and natural baroreceptor stimulation on nocicptive responding and pain. Psychophysiology 2003; 40:762-769.

Edwards L, McIntyre D, Carroll D, Ring C, Martin U. The human nociceptive flexion 
reflex threshold is higher during systole than diastole. Psychophysiology 2002; 39:678-681.

Edwards L, Ring C, McIntyre D, Carroll D. Modulation of the human nociceptive flexion reflex across the cardiac cycle. Psychophysiology 2001; 38:712-718.

Edwards L, Ring C, McIntyre D, Carroll D, Martin U. Psychomotor speed in hypertension: Effects of reaction time components, stimulus modality, and phase of the cardiac cycle. Psychophysiology 2007; 44:459-468.

Elbert T, Rockstroh B, Lutzenberger W, Kessler M, Pietrowsky R, Birbaumer N. Barorecepter stimulation alters pain sensation depending on tonic blood pressure. Psychophysiology 1988; 25:25-29.

Gahery Y, Vigier D. Inhibitory effects in cuneate nucleus produced by vago-aortic afferent-fibers. Brain Research 1974; 75:241-246.

Ghione S. Hypertension-associated hypalgesia. Hypertension 1996; 28:494-504.

Handwerker HO, Kobal G. Psychophysiology of Experimentally-Induced Pain. Physiological Reviews 1993; 73:639-671.

Inui K, Murase S, Nosaka S. Facilitation of the arterial baroreflex by the ventrolateral part of the midbrain periaqueductal grey matter in rats. Journal of Physiology 1994; 477:89-101. 
Kakigi R, Shibasaki H, Ikeda A. Pain-Related Somatosensory Evoked-Potentials Following Co2-Laser Stimulation in Man. Electroencephalography and clinical Neurophysiology 1989; 74:139-146.

Koch EB. Die Irradiation der pressorezeptorischen kreislaufreflexe. Klinische Wochenschrift 1932; 2:225-227.

Mancia G, Mark AL. Arterial baroreflexes in humans. In: Shepherd JT, Abboud FM, editors. Handbook of physiology. The cardiovascular system. Bethesda, Maryland: American Physiological Society, 1983. p. 755-793.

Mcallen RM. Identification and properties of sub-retrofacial bulbospinal neurons - A descending cardiovascular pathway in the cat. Journal of the Autonomic Nervous System 1986; 17:151-164.

McIntyre D, Edwards L, Ring C, Parvin B, Carroll D. Systolic inhibition of nociceptive responding is moderated by arousal. Psychophysiology 2006; 43:314-319.

Miller JF, Proudfit HK. Antagonism of stimulation-produced antinociception from ventrolateral pontine sites by intrathecal administration of $\alpha$-adrenergic antagonists and naloxone. Brain Research 1990; 530:20-34.

Mini A, Rau H, Montoya P, Palomba D, Birbaumer N. Baroreceptors cortical effects, emotions and pain. International Journal of Psychophysiology 1995; 19:67-77.

Mor J, Carmon A. Laser emitted radiant heat for pain research. Pain 1975; 1:233-237. 
Nakata H, Inui K, Wasaka T, Tamura Y, Tran TD, Qiu YH, Wang XH, Nguyen TB, Kakigi R. Movements modulate cortical activities evoked by noxious stimulation. Pain 2004; 107:91-98.

Peyron R, Frot M, Schneider F, Garcia-Larrea L, Mertens P, Barral FG, Sindou M, Laurent B, Mauguiere F. Role of operculoinsular cortices in human pain processing: Converging evidence from PET, fMRI, dipole modeling, and intracerebral recordings of evoked potentials. Neuroimage 2002; 17:1336-1346.

Rau H, Brody S, Larbig W, Pauli P, Vohringer M, Harsch B, Kroling P, Birbaumer N. Effects of PRES barorecepter stimulation on thermal an mechanical pain threshold in borderline hypertensives and normotensives. Psychophysiology 1994; 31:480-485.

Rau H, Elbert T. Psychophysiology of arterial baroreceptors and the etiology of hypertension. Biological Psychology 2001; 57:179-201.

Rau H, Elbert T, Lutzenberger W, Eves F, Rockstroh B, Larbig W, Birbaumer N. Pavlovian conditioning of peripheral and central components of the baroreceptor reflex. Journal of Psychophysiology 1988; 2:119-127.

Rau H, Pauli P, Brody S, Elbert T, Birbaumer N. Baroreceptor stimulation alters cortical activity. Psychophysiology 1993; 30:322-325.

Richter DW, Keck W, Seller H. Course of inhibition of sympathetic activity during 
various patterns of carotid sinus nerve stimulation. Pflugers Archiv-European Journal of Physiology 1970; 317:110-123.

Sandman CA, McCanne TR, Kaiser DN, Diamond B. Heart rate and cardiac phase influence on visual perception. Journal of Comparative and Physiological Psychology 1977; 91:189-202.

Sandman CA. Augmentation of the auditory event related potentials of the brain during diastole. International Journal of Psychophysiology 1984; 2:111-119.

Sandrini G, Serrao M, Rossi P, Romaniello A, Cruccu G, Willer JC. The lower limb flexion reflex in humans. Progress in Neurobiology 2005; 77:353-395.

Saxon SA. Detection of near threshold signals during four phases of the cardiac cycle. The Alabama Journal of Medical Sciences 1970; 7:427-430.

Scharein E, Bromm B. The intracutaneous pain model in the assessment of analgesic efficacy. Pain Reviews 1998; 5:216-246.

Seller H, Illert M. Localization of first synapse in carotid sinus baroreceptor reflex pathway and its alteration of afferent input. Pflugers Archiv-European Journal of Physiology 1969; 306:1-19.

Tarkka IM, Treede RD. Equivalent electrical source analysis of pain-related somatosensory-evoked potentials elicited by a Co2-Laser. Journal of Clinical Neurophysiology 1993; 10:513-519. 
Thurston CL, Randich A. Effects of vagal afferent stimulation on ON and OFF cells in the rostroventral medulla: Relationships to nociception and arterial blood pressure. Journal of Neurophysiology 1992; 67:180-196.

Thurston CL, Randich A. Responses to on and off cells in the rostral ventral medulla top stimulation of vagal afferents and changes in arterial pressure in intact and cardiopulmonary deafferentated rats. Pain 1995; 62:19-38.

Valeriani M, Rambaud L, Mauguiere F. Scalp topography and dipolar source modelling of potentials evoked by CO2 laser stimulation of the hand. Evoked Potentials-Electroencephalography and Clinical Neurophysiology 1996; 100:343-353.

Walker BB, Sandman CA. Visual evoked potentials change as heart rate and carotid pressure change. Psychophysiology 1982; 19:520-527.

Walker BB, Walker JM. Phase relations between carotid pressure and ongoing electrocortical activity. International Journal of Psychophysiology 1983; 1:65-73.

Wang X, Inui K, Kakigi R. Early cortical activities evoked by noxious stimulation in humans. Experimental Brain Research 2007; 180:481-489. 


\section{Figure captions}

Fig. 1. Pain-related evoked potential waveforms of 12 consecutive trials, depicting N2 and P2, in a representative participant.

Fig. 2. Mean (SE) N2-P2 peak-to-peak amplitudes as a function of phase of the cardiac cycle. A repeated measures ANOVA revealed significant variations in the N2-P2 amplitude across the cardiac cycle $(p=.02)$. Post-hocs confirmed that N2-P2 amplitudes elicited by stimulation at $\mathrm{R}+250, \mathrm{R}+350$ and $\mathrm{R}+450$ ms were smaller than those elicited at $\mathrm{R}+50, \mathrm{R}+150$ and $\mathrm{R}+750 \mathrm{~ms} . \mathrm{N}=10$, Trials $=45 . S E=S D \div \sqrt{ } \mathrm{N}$

Fig. 3. Grand average pain-related evoked potentials waveforms grouped into early ( $\mathrm{R}+50$ to $\mathrm{R}+150 \mathrm{~ms})$, middle $(\mathrm{R}+250$ to $\mathrm{R}+450 \mathrm{~ms})$, and late $(\mathrm{R}+550$ to $\mathrm{R}+750 \mathrm{~ms})$ phases of the cardiac cycle. $\mathrm{N}=10$, Trials $=45$.

Fig. 4. Mean (SE) N2 amplitudes as a function of phase of the cardiac cycle. A repeated measures ANOVA revealed significant variations in N2 amplitude across the cardiac cycle, $(p=.001)$. Post-hocs confirmed that $\mathrm{N} 2$ amplitudes elicited by stimulation at $\mathrm{R}+250$ ms was smaller than $\mathrm{R}+50, \mathrm{R}+150, \mathrm{R}+650$ and $\mathrm{R}+750$ ms. Stimulation at $\mathrm{R}+350$ ms produced smaller N2 amplitudes than $\mathrm{R}+150$, R+650 and R+750 ms. Finally, stimulation at $\mathrm{R}+450$ ms produced smaller $\mathrm{N} 2$ amplitudes than $\mathrm{R}+650$ and $\mathrm{R}+750 \mathrm{~ms}$. $\mathrm{N}$ $=10$, Trials $=45 . S E=S D \div \sqrt{ } \mathrm{N}$. 
Fig. 5. Mean (SE) P2 amplitudes as a function of phase of the cardiac cycle. A repeated measures ANOVA did not reveal significant variations in the P2 amplitude across the cardiac cycle $(p=.63) . \mathrm{N}=10$, Trials $=45 . S E=S D \div \sqrt{ } \mathrm{N}$

Fig. 6. Mean (SE) VAS pain ratings as a function of phase of the cardiac cycle. A repeated measures ANOVA revealed no significant differences in pain ratings across the cardiac cycle $(p=.37) . \mathrm{N}=10$, Trials $=45 . S E=S D \div \sqrt{ } \mathrm{N}$ 


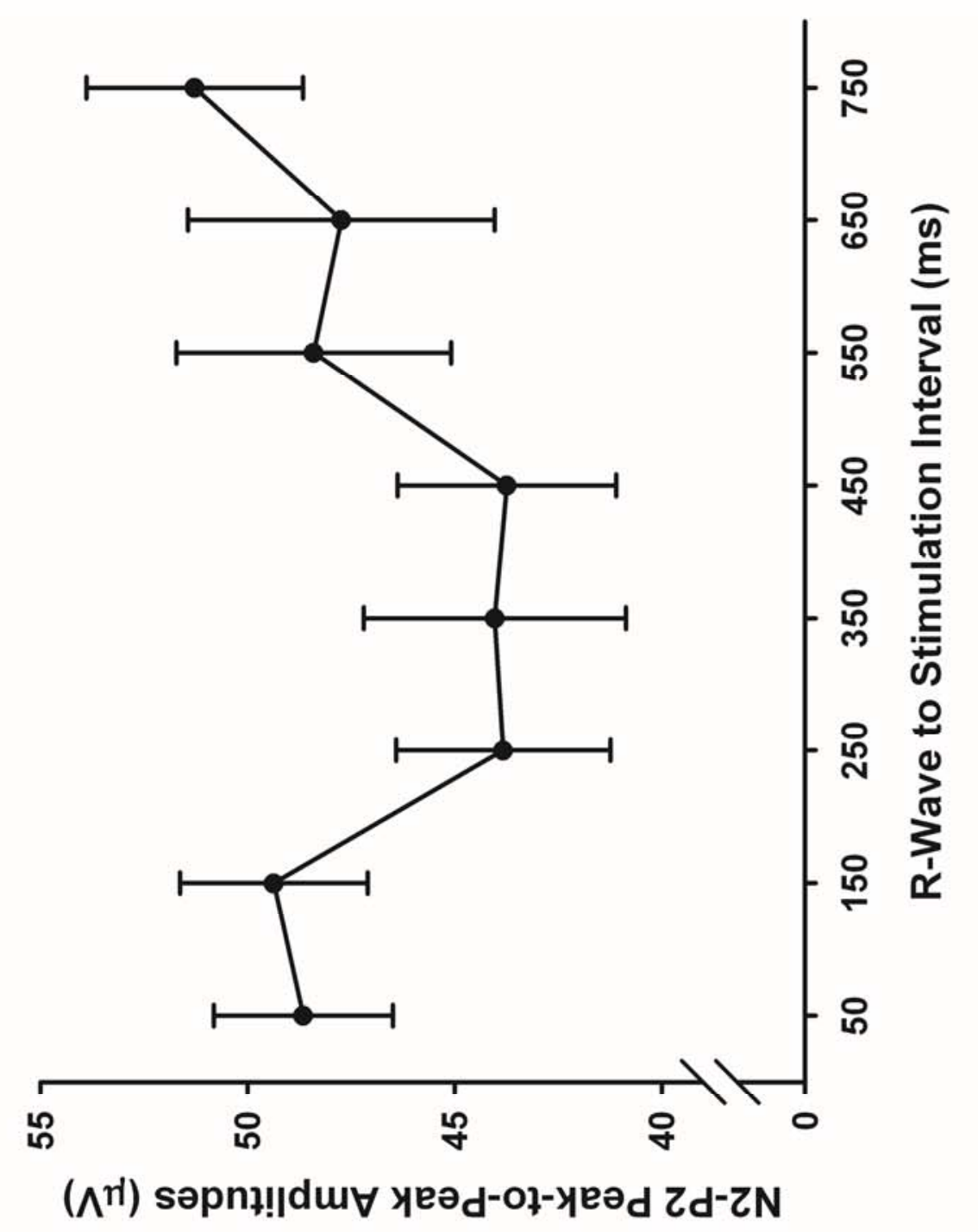




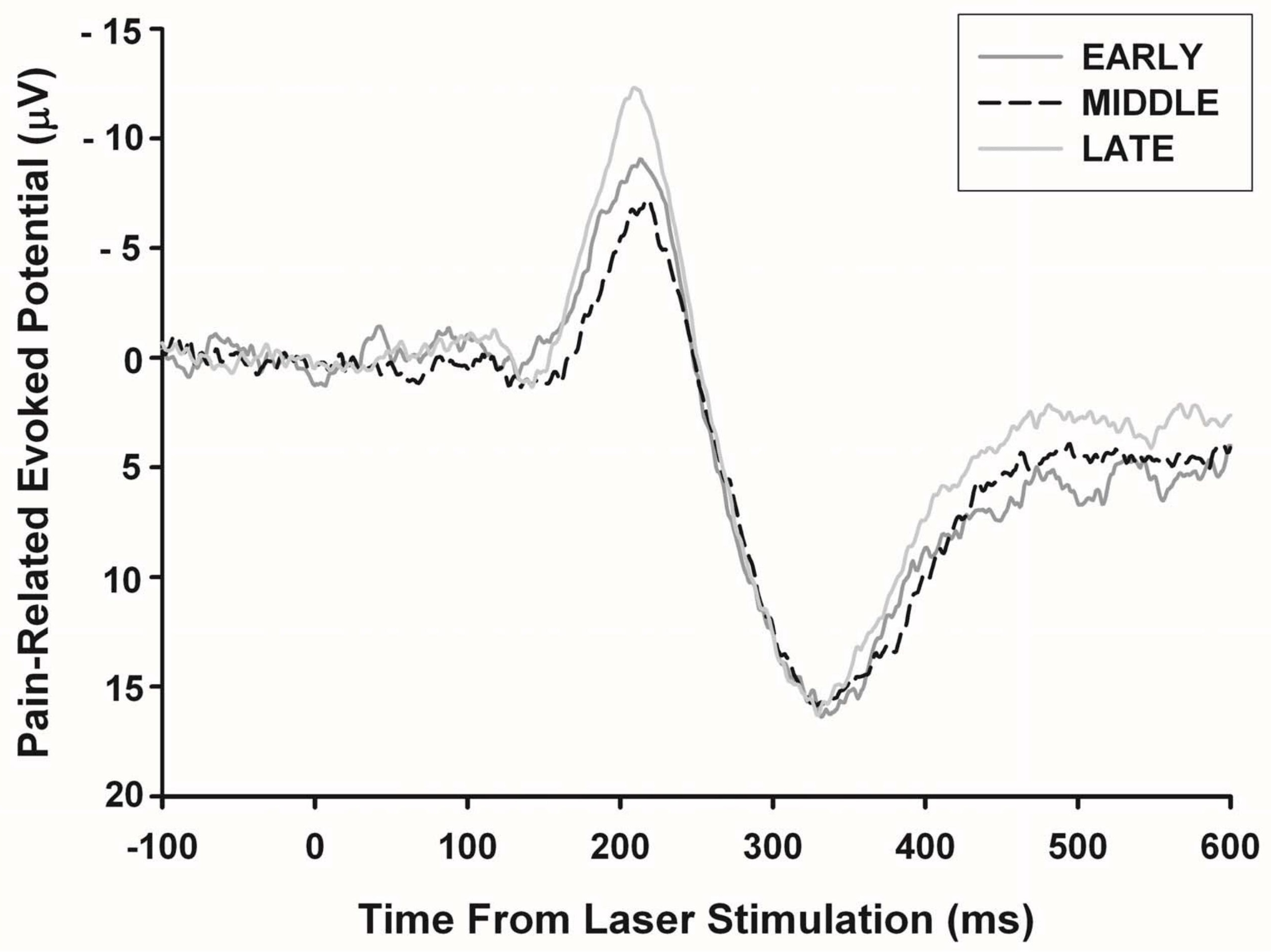




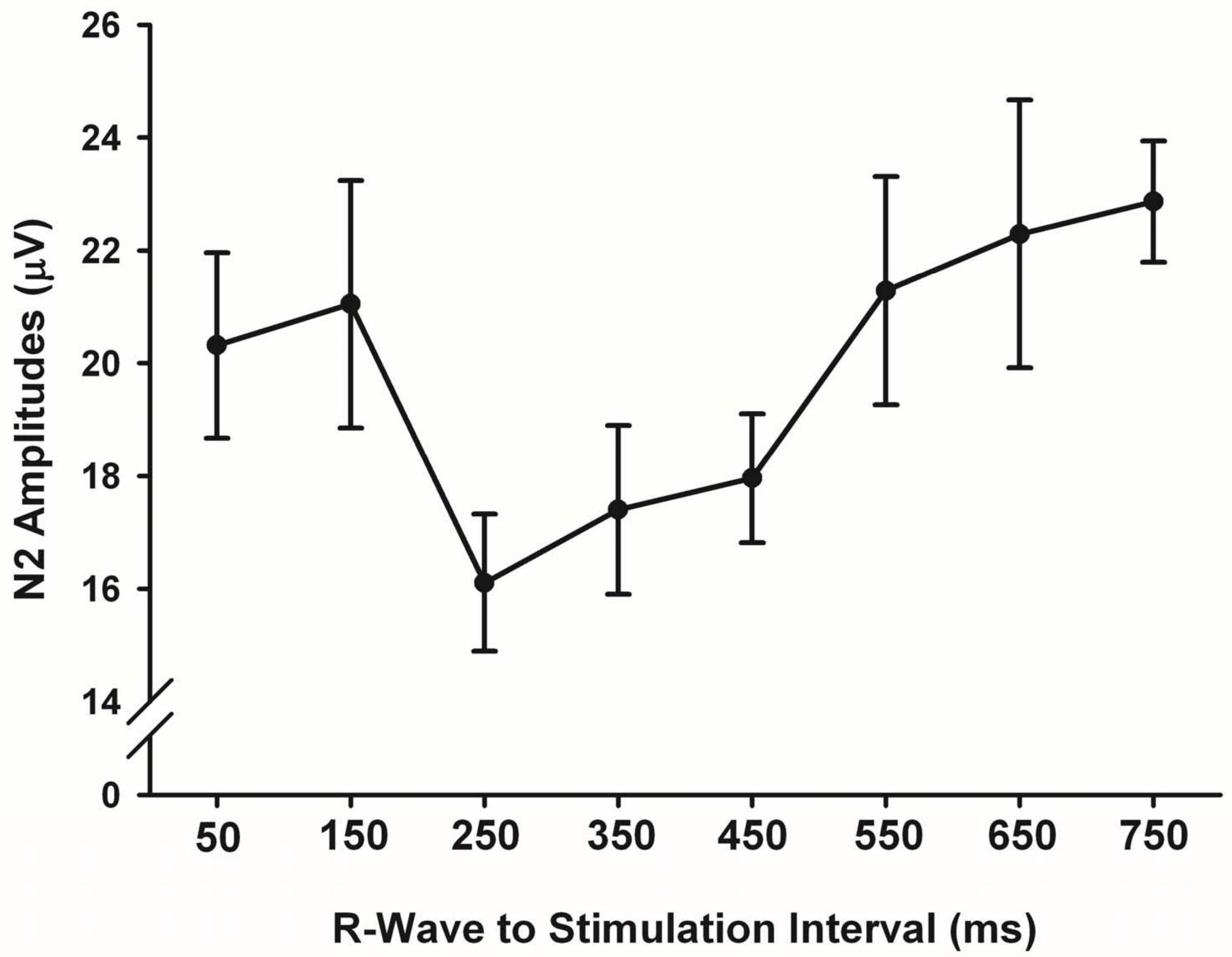




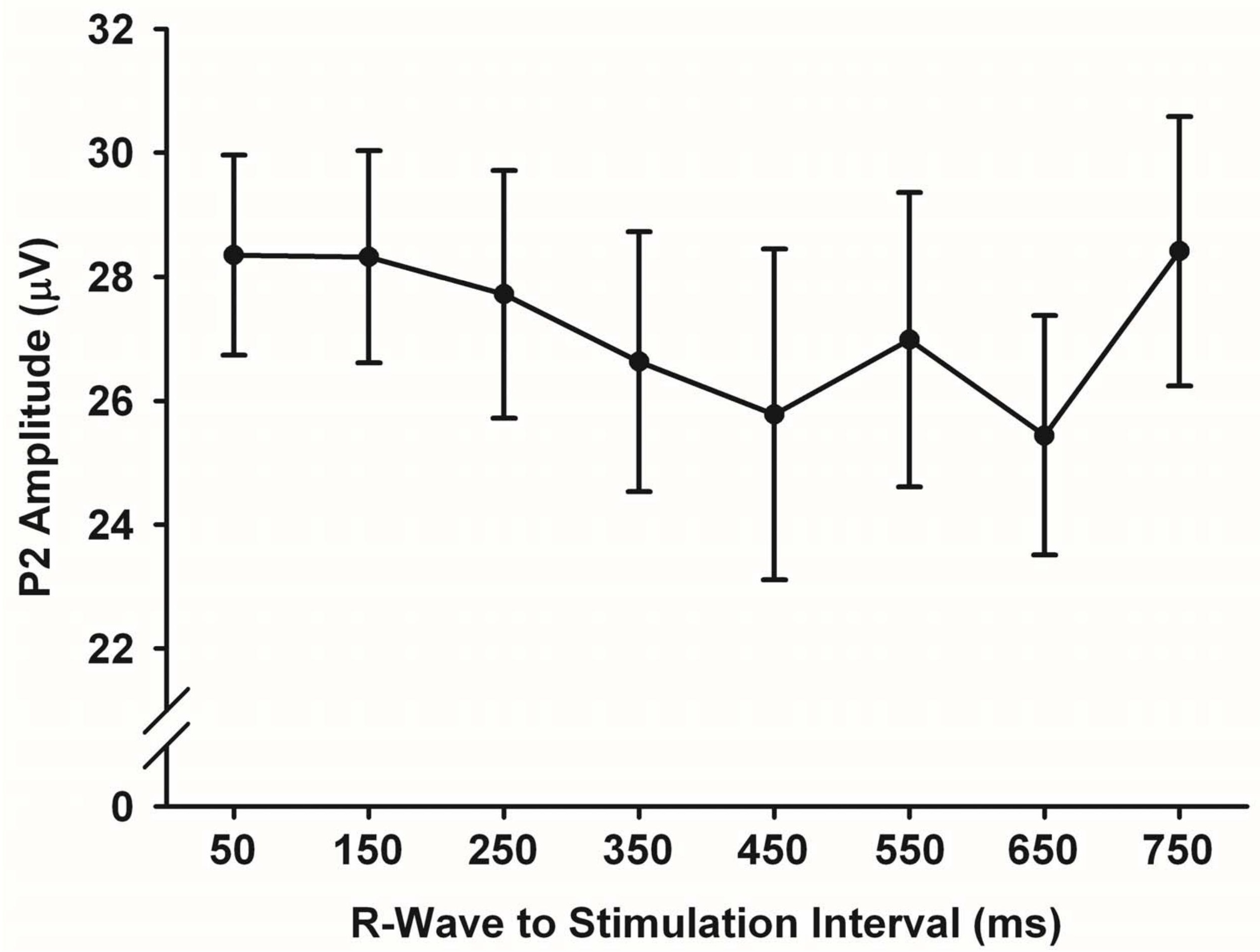




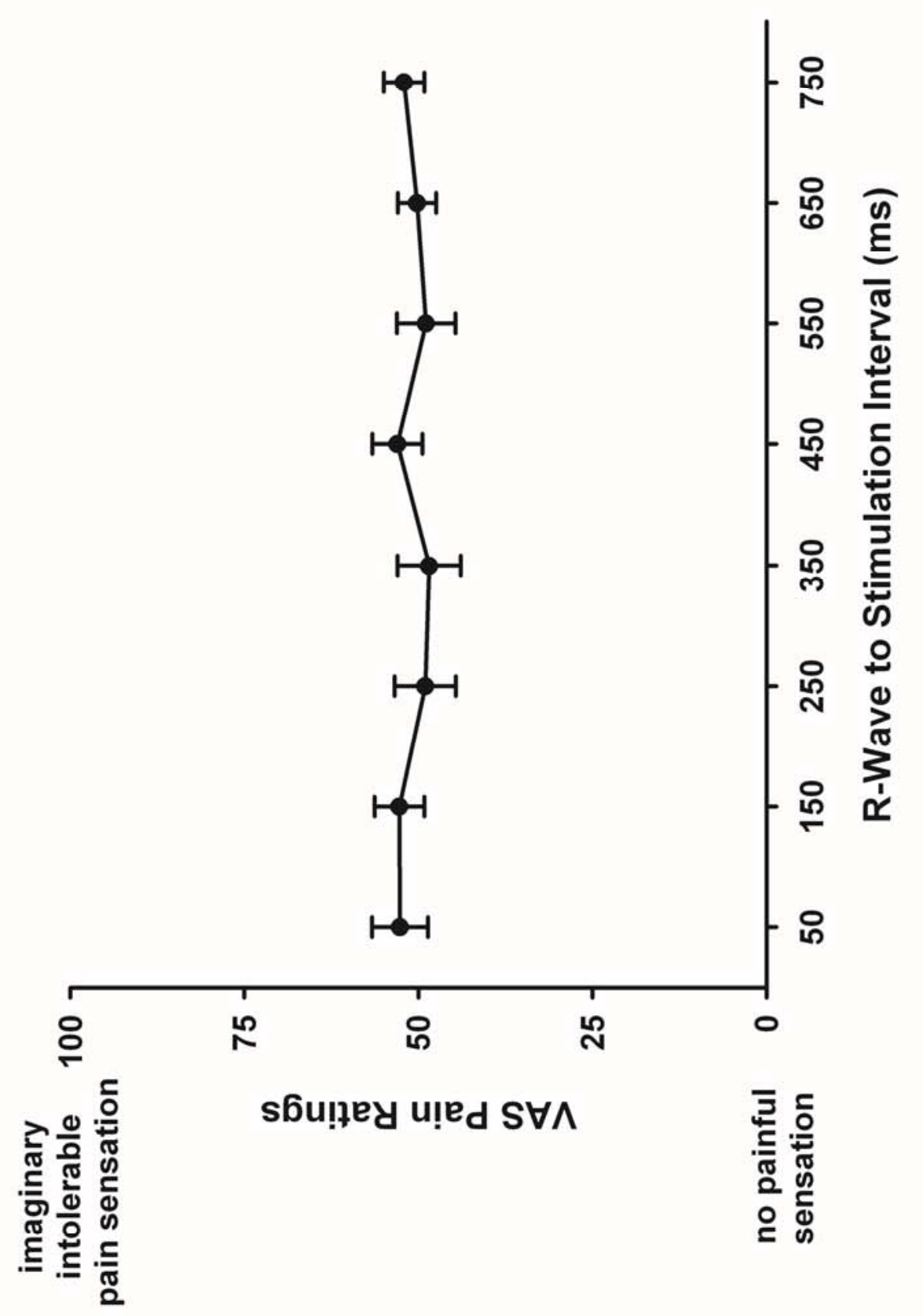

